\title{
Motor Cortex Bilateral Motor Representation Depends on Subcortical and Interhemispheric Interactions
}

\author{
Marcel Brus-Ramer, ${ }^{1 *}$ Jason B. Carmel, ${ }^{2 *}$ and John H. Martin ${ }^{1,3,4}$ \\ Departments of ${ }^{1}$ Neuroscience, ${ }^{2}$ Neurology, and ${ }^{3}$ Neurological Surgery and Psychiatry, Columbia University, and ${ }^{4}$ New York State Psychiatric Institute, \\ New York, New York 10032
}

The corticospinal tract is a predominantly crossed pathway. Nevertheless, the primary motor cortex (M1) is activated bilaterally during unilateral movements and several animal studies showed that M1 has a bilateral motor representation. A better understanding of the uncrossed corticospinal system is especially important for elucidating its role in recovery of limb control after unilateral injury. We used intracortical microstimulation (ICMS) to determine the representation of contralateral and ipsilateral forelimb joints at single M1 sites in the rat. Most sites representing an ipsilateral joint corepresented the same joint contralaterally. We next determined whether ipsilateral responses evoked in one hemisphere depended on the function of M1 in the opposite hemisphere using reversible inactivation and pyramidal tract lesion. Ipsilateral responses were eliminated when the homotopic forelimb area of M1 in the opposite hemisphere was inactivated or when the pyramidal tract on the nonstimulated side was sectioned. To determine the role of transfer between M1 in each hemisphere we sectioned the corpus callosum, which produced a 33\% increase in ipsilateral ICMS thresholds. Neither M1 inactivation nor callosal section changed contralateral response thresholds, indicating the absence of tonic excitatory or inhibitory drive to the opposite M1. Finally, ipsilateral responses following M1 inactivation and pyramidal tract lesion could be evoked after systemic administration of the $\mathrm{K}^{+}$channel blocker 4-aminopyridine, suggesting the presence of latent connections. Our findings show important interactions between the corticospinal systems from each side, especially at the spinal level. This has important implications for recruiting the ipsilateral corticospinal system after injury.

\section{Introduction}

The corticospinal tract (CST) is a predominantly crossed pathway. Emerging data, however, indicate that the primary motor cortex (M1) is activated bilaterally during production of unilateral movements (Donchin et al., 2002; Cisek et al., 2003). While much is known about the functions of the contralateral corticospinal (CS) system, much less is known about the ipsilateral component (Wang and Sainburg, 2006; Schaefer et al., 2007). A better understanding of the ipsilateral CST is especially important for elucidating its role in recovery after unilateral injury, when the only CS input to the hemiparetic side is the ipsilateral projection. This projection is implicated in motor recovery (Marshall et al., 2000; Weidner et al., 2001; Ward and Cohen, 2004; Brus-Ramer et al., 2007).

We used intracortical microstimulation (ICMS) of forelimb $\mathrm{M} 1$ in rats to address two key questions about the organization of the bilateral CS system. First, does the cortical representation of

Received Dec. 9, 2008; revised March 13, 2009; accepted March 16, 2009.

This work was supported by National Institutes of Health Grants NS36835, NS001698, and GM07367, and The New York State Spinal Injury Research Board Grant C022064. We acknowledge the assistance of XiuLi Wu for histoand immunohistochemistry, Dr. M. Osman for veterinary care, R. James Salway for help with experiments, Dr. Wendy Kartje for insightful discussion of our findings, and Drs. Kathleen Friel and Samit Chakrabarty for comments on this manuscript.

${ }^{*}$ M.B.-R. and J.B.C. contributed equally to this work.

Correspondence should be addressed to Dr. John H. Martin, Department of Neuroscience, Columbia University, 1051 Riverside Drive, New York, NY 10032. E-mail: jm17@columbia.edu.

DOI:10.1523/JNEUROSCI.5852-08.2009

Copyright $\odot 2009$ Society for Neuroscience $\quad$ 0270-6474/09/296196-11\$15.00/0 the ipsilateral forelimb mirror that of the contralateral representation, or do they have different organizations? Whereas earlier rat studies demonstrated a bilateral M1 forelimb motor representation (Kartje-Tillotson et al., 1985; Liang et al., 1993), the relationship between contralateral and ipsilateral responses evoked at single M1 sites is not well understood. An ipsilateral representation that mirrors the contralateral representation would be well suited to help restore movements after injury.

Second, do bilateral responses evoked by ICMS in one hemisphere depend on the CS system from the other hemisphere? The ipsilateral M1 projection is sparse (Brösamle and Schwab, 1997) and weak (Edgley et al., 2004; Jankowska et al., 2005; Brus-Ramer et al., 2007). Motor circuits on the side receiving this sparse ipsilateral CST projection also receive dense inputs from M1 in the other hemisphere (see Fig. 1). The ipsilateral projection may need a background of CS activation from the other hemisphere to contribute to control. This organization may help to integrate the motor functions of the two hemispheres. To determine whether ipsilateral responses depend on the crossed descending M1 projections, we used reversible M1 inactivation and pyramidal tract (PT) lesion to depress CS system function from one side while the other (intact) M1 was mapped. To determine the importance of interhemispheric interactions, we cut the corpus callosum. We further tested whether changes in ipsilateral motor responses produced by inactivation or PT lesion could be reversed by elevating CS system efficacy using the $\mathrm{K}^{+}$channel blocker, 4-aminopyridine (4-AP) (Jankowska et al., 1982). 4-AP, among other effects (Nashmi and Fehlings, 2001), enhances synaptic 
transmission of descending cortical axons (Jankowska et al., 2005).

We found remarkable overlap of the ipsilateral and contralateral maps in each hemisphere. ICMS at the same site also produced similar distal or proximal joint responses, suggesting mirroring of the two representations. Surprisingly, we found that ipsilateral responses depended on the contralateral CS system, through moderate interhemispheric facilitation and strong subcortical facilitation, and that this dependence was abrogated by 4-AP. Our findings suggest that the ipsilateral CS system in the rat has latent connection with properties making it well suited to restore CS function after unilateral injury.

\section{Materials and Methods}

Experiments were conducted on adult female Sprague Dawley rats $(n=$ $26 ;>225 \mathrm{~g}$ ). All procedures were approved by the institutional animal use committees of Columbia University and the NYS Psychiatric Institute.

Intracortical microstimulation. For ICMS motor mapping, anesthesia was induced with ketamine ( $80 \mathrm{mg} / \mathrm{kg}$, i.p.) and xylazine $(10 \mathrm{mg} / \mathrm{kg}$, i.p.) and maintained using intraperitoneal ketamine injections to render the animal unresponsive to paw pinch. Ketamine anesthesia maintains muscle tone and therefore is well suited for motor mapping studies. Animals were placed in a stereotaxic frame. Body temperature was maintained at $39^{\circ} \mathrm{C}$ by a heating pad. A craniotomy was made over the forelimb area of M1. Electrode penetrations, perpendicular to the pial surface and $\sim 0.5$ $\mathrm{mm}$ apart, were made into M1. In all animals, the region sampled was the same, from 1.5 to $4.5 \mathrm{~mm}$ lateral to bregma and -0.5 to $5.5 \mathrm{~mm}$ rostral to bregma. We used low impedance tungsten microelectrodes (Microprobe; 0.5 MÙ impedance; $0.081 \mathrm{~mm}$ shaft diameter, $1-2 \mu \mathrm{m}$ tip diameter). Motor effects produced by microstimulation occurred at lowest stimulus currents, at the same depths where we recorded multiunit activity with the largest amplitude spikes (typically $1.1-1.3 \mathrm{~mm}$ below the pial surface). Marking lesions were made to help identify the location of the region sampled. For ICMS experiments without callosal axon tracing, the sampled cortical area was removed and postfixed in $10 \%$ formalsaline. Sections were cut and Nissl stained.

Stimuli ( $45 \mathrm{~ms}$ duration train, $330 \mathrm{~Hz}, 0.2 \mathrm{~ms}$ biphasic; every $2 \mathrm{~s}$ ) were delivered using a constant current stimulator (A-M Systems). The threshold is defined as the lowest current that consistently produced a motor effect ( $>50 \%$ of trials). For a given site, we started at a low current and first determined the threshold for evoking a contralateral response. Then, as current amplitude increased, the ipsilateral response threshold was determined. The thresholds were then examined in reverse through the loss of the responses with decreasing currents. As we obtained the motor map within M1, we randomized placement of the electrode to prevent biasing our results by anesthesia level or other state-dependent changes. A maximal current of $100 \mu \mathrm{A}$ was used systematically. However, after contralateral cortex inactivation or pyramidotomy, which abrogated ipsilateral responses (see below), we occasionally used higher currents at previously effective sites so as not to miss a response. For each penetration, the type of motor effect produced by a threshold and 1.5 times threshold stimulus was determined on the basis of the evoked phasic kinematic change; adjacent joints were stabilized. We tallied the number of sites evoking movements and computed the effective area, noting current threshold and sites evoking movement at multiple joints. Limb posture was the same for all experiments; with the shoulder and elbow extended, and the wrist flexed. To determine whether the efficacy of ipsilateral CS responses could be augmented, we administered 4-AP (Sigma; $2 \mathrm{mg}$ dissolved in saline/kg, i.p.).

Reversible inactivation. To determine whether the ipsilateral motor map in one M1 depends on the activity of M1 in the other cortex, one M1 was mapped and the lowest threshold point (or several neighboring points) was used to stimulate before, during, and after reversible inactivation of the other cortex. In each animal, a complete motor map was obtained before the reversible inactivation experiment began. After identifying the lowest threshold point on one side, motor response thresholds were determined for the equivalent point and nearby points in the oppo- site hemisphere. Next, a $2 \%$ solution of lidocaine $(1 \mu \mathrm{l})$ tinted with Evan's Blue (Sigma) was pressure injected $(200 \mathrm{nl} / \mathrm{min})$ into the hemisphere opposite the one more thoroughly mapped, $1.5 \mathrm{~mm}$ below the brain surface at the stereotaxic position equivalent to the lowest threshold point. Tinted lidocaine was injected using a glass micropipette (25 $\mu \mathrm{m}$ tip diameter) connected to a Hamilton syringe and an infusion pump (Harvard Apparatus). Evans blue fluoresces in the Cy3 range (570 $\mathrm{nm}$ ). We used this fluorescence within the injected cortex as an estimate of drug spread (see supplemental Fig. 2, available at www.jneurosci.org as supplemental material). In one animal, motor response thresholds were recorded from the original low threshold position in the un-injected hemisphere every minute over a $15 \mathrm{~min}$ period to determine the onset of effects from lidocaine infusion at a homotopic position in the alternate cortex. In three animals, thresholds were recorded at the low threshold position for the uninjected hemisphere once every $5 \mathrm{~min}$ for the first 20 $\mathrm{min}$, and then once every $10 \mathrm{~min}$ for the remainder, until microstimulation thresholds recovered to baseline values (60-75 $\mathrm{min})$. Additionally, to confirm lidocaine inactivation, we noted the absence of motor responses from the injected hemisphere. After the thresholds for ipsilateral and contralateral responses returned to baseline, additional nonhomotopic lidocaine injections were made. In two animals, a lidocaine injection ( $1 \mu \mathrm{l}$ at $200 \mu \mathrm{l} / \mathrm{min}$ ) was made in the hind limb (3.5 mm caudal to the initial injection) motor area (Neafsey et al., 1986) of the previously injected hemisphere. In a second animal a non-homotopic injection was made $3.5 \mathrm{~mm}$ rostral to the original injection, a position anterior to the rostral forelimb area (Neafsey et al., 1986).

Callosal axon tracing. To determine the location of M1 interhemispheric axons in intact animals and after callosal section, we used the short survival-time anterograde tracer Neurobiotin. After mapping, we pressure-injected $300 \mathrm{nl}$ of a 15\% solution of Neurobiotin (Vector Labs) at a depth of $1.5 \mathrm{~mm}$ from the pial surface at the center of the region containing lowest thresholds ( $n=2$ controls). An additional injection was then made at each of the four positions immediately surrounding (0.5 $\mathrm{mm}$ distance) this site. After the injections, the craniotomy was covered with gel foam and the overlying skin closed, and the animals replaced to their cage. After $12 \mathrm{~h}$, the brain was reexposed and marking lesions were made at 3 rostrocaudal positions $4.5 \mathrm{~mm}$ lateral to bregma with a $25 \mathrm{~g}$ needle: bregma, $2 \mathrm{~mm}$ rostral, and $2 \mathrm{~mm}$ caudal to bregma. Immediately, after the marking lesions were made, the animals were perfused to determine the locations of labeled callosal axons relative to key anatomical landmarks. See below for Neurobiotin histochemistry.

Corpus callosum section. The anterior corpus callosum, where axons connecting the motor cortices cross, was sectioned to help determine the locus of interaction between the two halves of the CS system. The site and depth of callosal sections were determined on the basis of the results of our tracing experiments described above with reference to a stereotaxic atlas (Paxinos and Watson, 1986). For these experiments, bregma coordinates were obtained and a craniotomy was made that extended to the midline. Lesions were made either with a $2 \mathrm{~mm}$ wire knife (diameter of knife arc; Kopf Instruments) or a \#11 scalpel blade; each were held in a stereotaxic arm. The initial penetration was made $1.8 \mathrm{~mm}$ rostral to bregma and extended anteriorly until all of the anterior callosal fibers were cut. Care was maintained to preserve the integrity of the sagittal sinus and any crossing blood vessels. We verified that the lesion interrupted M1 interhemispheric connections by injecting Neurobiotin before sectioning the corpus callosum ( $n=5$ rats). We also verified that the corpus callosum was sectioned, sparing underlying structures, by examining Nissl-stained sections of postmortem tissue.

Pyramidal tract lesion. PT lesions were made for several purposes: to determine the effect of eliminating the CS projection on ipsilateral (1) and contralateral (2) responses evoked by ICMS on the nonlesioned side, and to determine whether enhancing weak ipsilateral CS connections using 4-AP restored ipsilateral responses after the lesion. A small craniotomy $(2 \times 2 \mathrm{~mm})$ was made in the ventral surface of the occipital bone exposing the PT on the ventral brainstem surface. For PT lesion, a pair of fine-tip forceps (Fine Science) were lowered to a depth of $1.1 \mathrm{~mm}$ below the ventral medulla surface $[13 \mathrm{~mm}$ caudal to bregma (Paxinos and Watson, 1986)] immediately adjacent to the basilar artery. The forceps were used to cut axons in the pyramidal tract and separate the cut ends, 
ensuring a complete lesion of one pyramidal tract. Lesions were verified histologically by transverse sections at the lesion site (supplemental Fig. 1, available at www.jneurosci.org as supplemental material). When the PT lesion was made during an experiment, the cortex was covered with gel foam and the skin apposed with wound clips to protect the cortex from damage. After completion of the lesion surgery, the pyramid was covered with gel foam and the skin apposed with wound clips, the animal is placed in the frame, and new coordinates for bregma were obtained. For survival experiments, M1 was mapped after the lesion only. We stimulated M1 on the intact side and determined that the threshold for evoking contralateral movements had not changed as a consequence of these manipulations.

Tissue preparation and staining. For the combined ICMS and callosal tracing experiments, the animals were given an anesthetic overdose and perfused with saline followed by $4 \%$ paraformaldehyde. The brain was dissected, postfixed for $2 \mathrm{~h}$ and then transferred to $20 \%$ sucrose in buffered water, $\mathrm{pH}$ 7.4. For the lidocaine inactivation experiments, animals were killed by anesthetic overdose. Brains were quickly removed and postfixed with $4 \%$ paraformaldehyde for $2 \mathrm{~d}$ at $4^{\circ} \mathrm{C}$, and then transferred to $20 \%$ sucrose in buffer. Frozen sections $(40 \mu \mathrm{m})$ were cut serially and collected in a 0.1 $\mathrm{M}$ PBS solution, $\mathrm{pH}$ 7.4. For examining the topography of callosal axons, we used nickel intensification of diaminobenzidine $(\mathrm{DAB})$ reaction product, using the avidin-biotin complex (ABC) method (Vector Laboratories) according to the manufacturer's instructions. Sections were washed in $0.1 \mathrm{~m}$ PBS and incubated in the $\mathrm{ABC}$ reagent-Triton solution (made $30 \mathrm{~min}$ before use: two drops of A and two drops of B in 5 $\mathrm{ml}$ of $0.2 \%$ Triton in $0.1 \mathrm{M}$ PBS) for $5-6 \mathrm{~h}$ at room temperature or overnight at $4^{\circ} \mathrm{C}$. The tissue was washed in $0.1 \mathrm{M}$ PBS and then for 10 min in $0.01 \mathrm{~m}$ sodium acetate $(\mathrm{NaAc})$. The tissue was incubated in $\mathrm{DAB}$, intensified using nickel ammonium sulfate [ 1 tablet of DAB, 10 $\mathrm{ml}$ of NaAc, $10 \mathrm{ml}$ of nickel ammonium sulfate solution (5.5 g of nickel ammonium sulfate in $100 \mathrm{ml}$ of $\mathrm{NaAc}$ ), $12 \mathrm{ml}$ of $30 \% \mathrm{H}_{2} \mathrm{O}_{2}$ ]. The sections were incubated in this solution for 6-30 min. The sections were washed once for $10 \mathrm{~min}$ in a $0.01 \mathrm{M}$ $\mathrm{NaAc}$ solution and twice for $10 \mathrm{~min}$ each in a $0.1 \mathrm{~m}$ PBS solution. Selected sections were Nissl stained.

EMG recording and analysis. We recorded electromyographic (EMG) responses from forelimb muscles using percutaneous $\mathrm{Ni}$-chrome wire electrodes and a differential AC amplifier with low and high pass filtration (A-M Systems). The skin was shaved over the forelimbs to facilitate recording electrode placement. EMG recording wires were inserted into a 26-gauge hypodermic needle. They were deinsulated at the tip (1 mm) and a small hook was formed by bending the wire over the needle tip. The needle-wire assembly was inserted into the muscle and the needle withdrawn, leaving the wire securely embedded within the muscle. We recorded differentially, with two wire electrodes within each muscle. The ground electrode was placed at a distant site. We verified muscle placement by reflex testing. For a flexor muscle (e.g., biceps), the joint distal to the muscle was extended to evoke a stretch reflex-evoked burst or tonic increase in activity. For an extensor muscle, the joint was passively flexed. EMG signals were acquired using an analog-to-digital converter (Digidata; Axon Instruments) at $20 \mathrm{kHz}$ per channel and processed using the program AxoGraph for the Apple Macintosh computer. For analysis and display, EMGs were first rectified and then averaged. Recordings were synchronized with the onset of cortical stimulation.

Statistical analyses. Standard statistical tests including Student's $t$ test and ANOVA were conducted using Microsoft Excel (Microsoft) and JMP 7 (SAS Institute).

\section{Results}

We investigated the extent of the ipsilateral forelimb representation in M1 and its dependence on the function of M1 in the other hemisphere. Initial experiments using ICMS of one hemisphere ( $n=4$ rats) mapped the representations of the ipsilateral and contralateral forelimbs and the types of evoked responses in each forelimb (Fig. 1, right). Reversible inactivation of the nonstimulated M1 ( $n=8$ rats) and lesion of the PT on the nonstimulated side ( $n=6$ rats) were used to determine whether an active and functioning CS system opposite the stimulated M1 was necessary for evoking ipsilateral responses (Fig. 1). Corpus callosal section ( $n=8$ rats) was used to determine whether ICMS evoked ipsilateral responses through interhemispheric activation of the opposite M1 (Fig. 1, blue callosal axon). Finally, we used systemic admin- 
A1. Contralateral

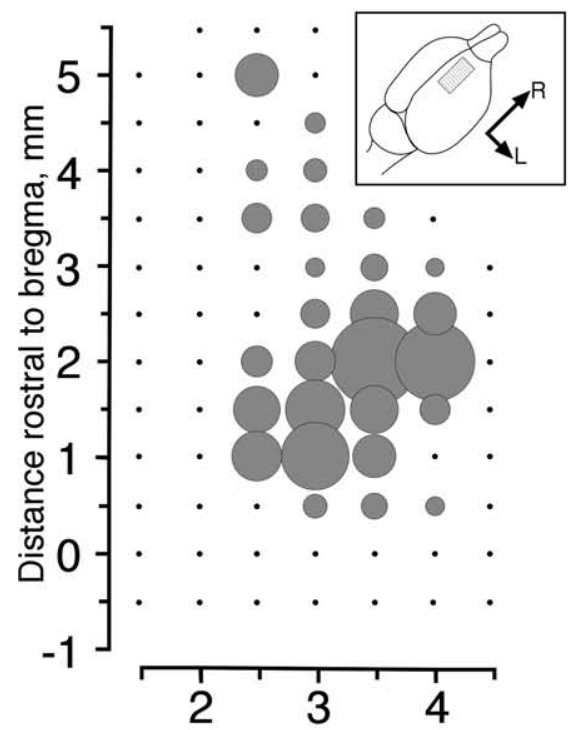

\section{A2. Contralateral}

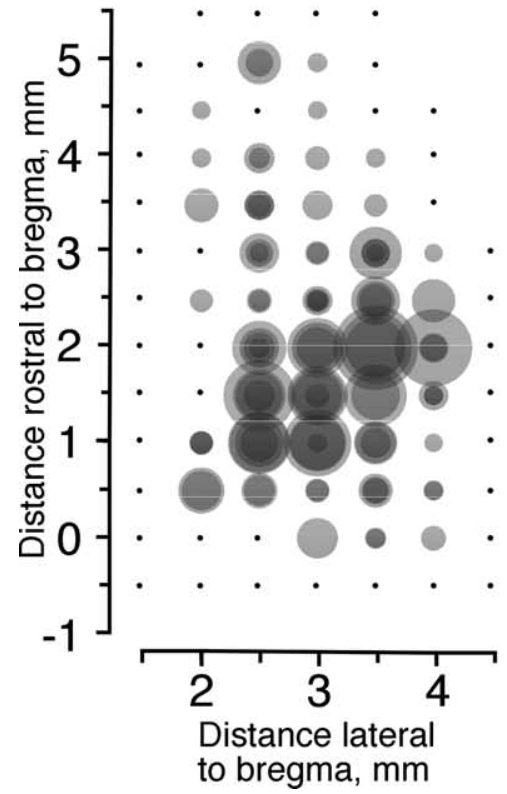

B1. Ipsilateral

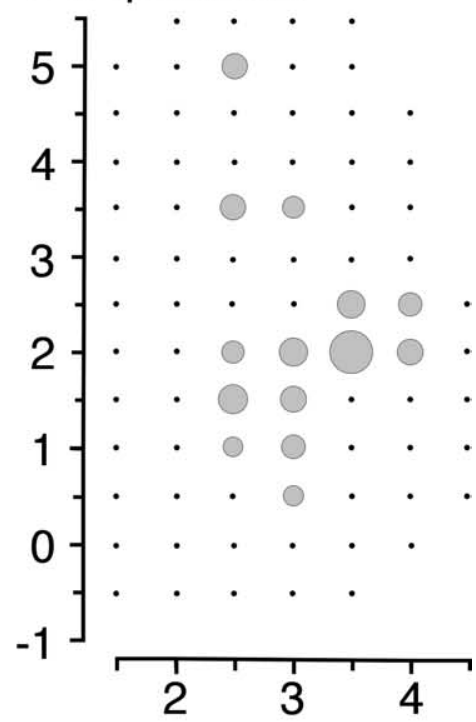

B2. Ipsilateral

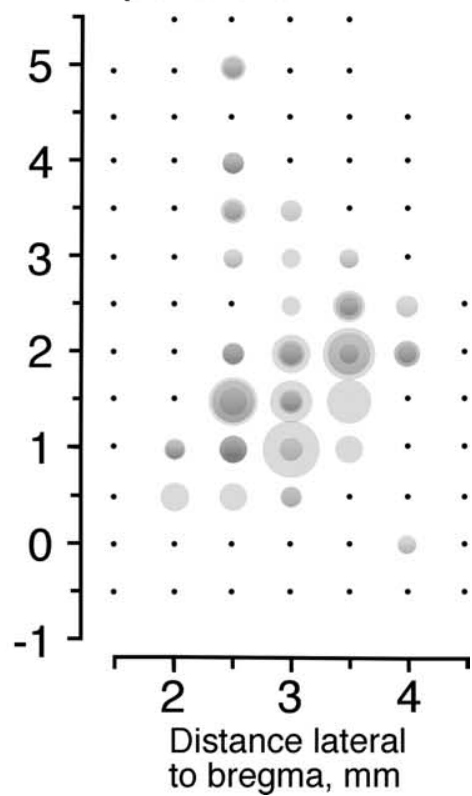

Figure 2. The ipsilateral and contralateral forelimb representations are coextensive. Bubble plots of contralateral (left) and ipsilateral (right) forelimb responses evoked from the forelimb area of $\mathrm{M} 1$ in a single representative normal animal $(\boldsymbol{A 1}, \boldsymbol{B 1})$ and in normal animals $(n=4)$. Bubble size is inversely related to the threshold for evoked forelimb response (1 per threshold in microamperes). Bubble opacity in $\boldsymbol{A} \mathbf{2}$ and $\boldsymbol{B} \mathbf{2}$ represents percentage of electrode penetrations evoking a response at that site. Points with black dots are sites where no response was evoked using currents up to $100 \mu \mathrm{A}$. All electrode penetration sites are separated from adjacent penetrations by $500 \mu \mathrm{m}$ distance in rostrocaudal or mediolateral directions. The inset shows a rat brain and the approximate location of the region mapped ( $R$, rostral; $L$, lateral).

istration of 4-AP to determine whether we could reestablish ipsilateral ICMS responses in animals with M1 lidocaine inactivation $(n=2)$ or PT lesion of the other half of the CS system $(n=4)$.

\section{The ipsilateral and contralateral forelimb representations} are coextensive

We mapped the rostral and caudal forelimb representations (Neafsey et al., 1986) between 0.0 and $5.5 \mathrm{~mm}$ rostral to bregma and 1.5 and $4.5 \mathrm{~mm}$ lateral, by determining the sites that evoked limb movements, the laterality of the movements, and which joints moved ( $n=4$ animals). To standardize results, we maintained the forelimbs in a consistent posture, with the wrist flexed and the elbow and shoulder extended. We found that ipsilateral responses were more readily evoked when limb muscles were stretched in this posture. Figure 2, $A 1$ and $B 1$, shows results of mapping contralateral and ipsilateral effects from one representative animal; Figure 2, $A 2$ and $B 2$, presents results from the mapped hemisphere in four animals. Each circle plots the inverse threshold (1 per current in microamperes) for evoking a forelimb response at each effective site, i.e., larger circles indicate motor responses at lower current. Black dots mark sites that were ineffective at evoking forelimb responses. For Figure $2 B$, response thresholds from each animal are overlaid, so darker gray indicates greater reproducibility among the animals tested. The contralateral and ipsilateral forelimb representations were coextensive and the sites with the lowest contralateral and ipsilateral thresholds overlapped. The contralateral representation comprised an area of $6.1 \pm$ $0.7 \mathrm{~mm}^{2}$ (24.5 \pm 2.6 sites per animal), and the ipsilateral representation, $3.1 \pm 0.5 \mathrm{~mm}^{2}$ $(12.3 \pm 2.2$ sites per animal).

We also compared sites evoking bilateral responses with those evoking only unilateral responses. Sites evoking bilateral responses were as common as contralateral only sites (bilateral: $50.8 \%$; contralateral only: $49.2 \%$ ), similar to other reports in the rat (Kartje-Tillotson et al., 1985; O’Donoghue et al., 1986). At all sites tested, the contralateral thresholds were lower than ipsilateral thresholds (contralateral mean, $45.3 \pm 4.4 \mu \mathrm{A}$; ipsilateral mean, $62.0 \pm 8.9 \mu \mathrm{A})$. At sites producing bilateral responses (i.e., 50.8\%), average contralateral thresholds were significantly lower than ipsilateral responses (contralateral, $25.8 \pm 2.9 \mu \mathrm{A}$; ipsilateral, $61.0 \pm 8.9$ $\mu \mathrm{A}$; paired $t$ test $p$ values for each animal: $0.00013,0.00011,0.0000002,0.000013$ ). Finally, contralateral thresholds at sites evoking bilateral responses were significantly lower than at contralateral only sites $(25.8 \pm 2.9 \mu \mathrm{A}$ vs $64.2 \pm 5.3 \mu \mathrm{A}$; paired $t$ test, $p=0.006)$. This suggests that the bilateral sites in M1 have the strongest connections with contralateral spinal motor circuits. Our findings show that the spatial extent and stimulation responsiveness of the ipsilateral forelimb representation is approximately half that of the contralateral forelimb representation.

The ipsilateral and contralateral maps corepresent the same forelimb joints

ICMS evoked digit flexion, wrist extension, elbow flexion, and/or shoulder flexion or elevation, alone, or in combination. This is 


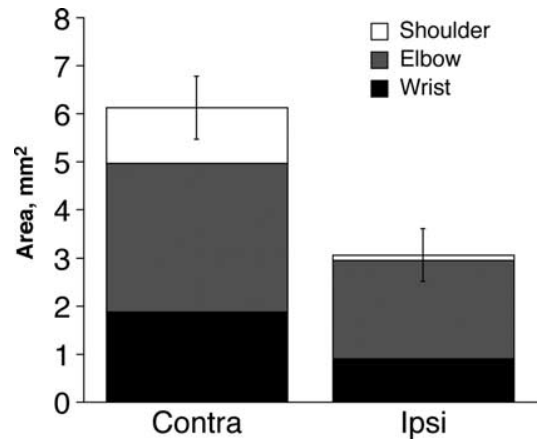

Figure 3. Size and distribution of forelimb joints in the contralateral and ipsilateral representations. Bar graphs plot the total area of the contralateral and ipsilateral representations. The ipsilateral forelimb representation has a largely similar distribution of joint types as the contralateral. The SE bars plot between animal variability of total forelimb M1 representation area.

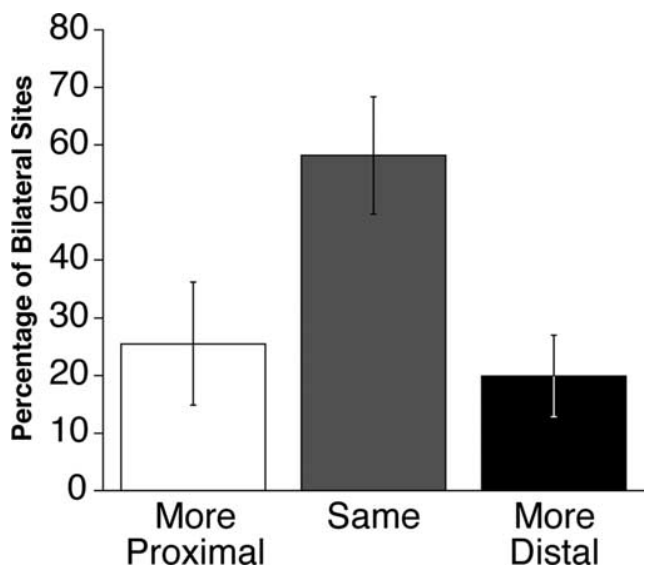

Figure 4. At bilateral sites, ipsilateral and contralateral responses are predominantly at the same joint. Bar graphs plot the ipsilateral response relative to the contralateral response at sites where both were evoked. At the majority of bilateral sites $(58.2 \pm 10.2 \%)$, the evoked ipsilateral forelimb responses occurred at the same joint as the evoked contralateral responses.

similar to a previous study (Sanes et al., 1992). Other responses (e.g., elbow extension, digit extension) were rarely, if ever, observed. Stimulation at $30.5 \pm 4.2 \%$ of sites evoked more than one contralateral response within 1.5 times threshold (i.e., multijoint sites) and $18.0 \pm 4.0 \%$ of sites evoked more than one ipsilateral response. For either the contralateral or ipsilateral responses, movement at one joint typically was larger than those of the other joints. Figure 3 presents the area of cortex where motor responses are produced for the different forelimb joints. For the purpose of this analysis, responses evoked at multijoint sites were recorded as the dominant response. Digits are not shown because wrist responses dominated at those multijoint sites. Contralateral and ipsilateral motor responses were predominantly elbow flexion and wrist extension, similar to a previous report (Neafsey et al., 1986). These findings suggest that the ipsilateral M1 forelimb representation, like the contralateral representation, is largely for distal control.

We next determined whether ICMS sites evoking bilateral responses produced the same response in each forelimb or if the ipsilateral movements were proximal or distal to the contralateral ones. Cortical sites where ipsilateral and contralateral responses were evoked produced the same joint response at $58.2 \pm 10.2 \%$ of the sites (Fig. 4). In $19.9 \pm 7.1 \%$ of the sites the ipsilateral responses were more distal, and in $25.5 \pm 10.6 \%$ of the sites, more proximal. Thus, the majority of sites in $\mathrm{M} 1$ from which ipsilateral

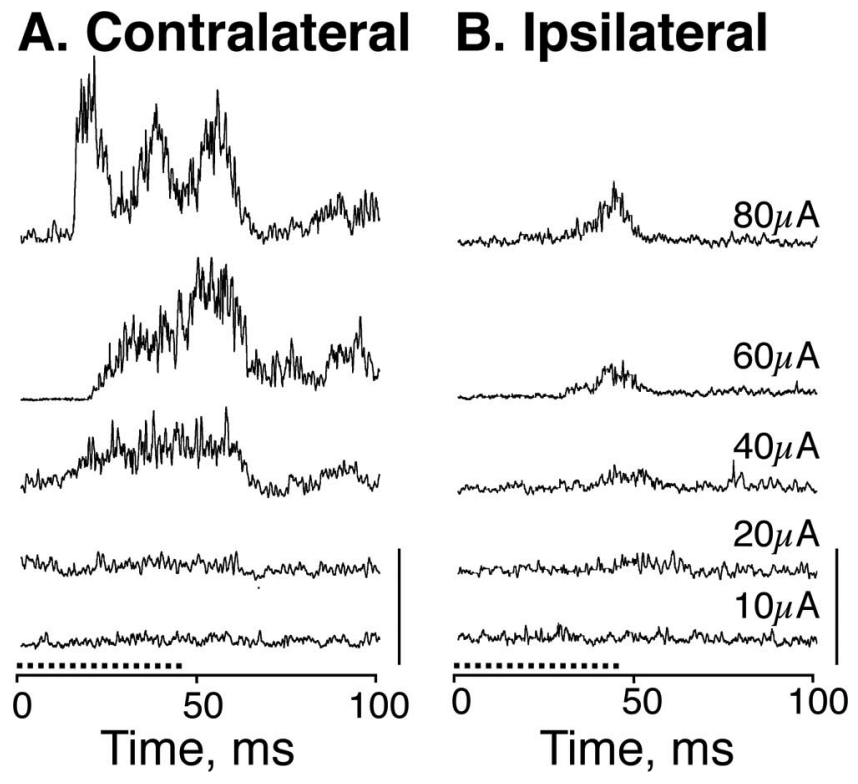

Figure 5. EMG correlates with thresholds for movement. Ensemble averages ( $n=20$ stimuli) of contralateral $(\boldsymbol{A})$ and ipsilateral $(\boldsymbol{B})$ biceps EMG recorded simultaneously during ICMS at five different current levels. The stimulus threshold for evoking contralateral elbow flexion was $15 \mu \mathrm{A}$ and $22 \mu \mathrm{A}$ for ipsilateral elbow flexion. The dotted lines indicate the simulation period (45 ms). Calibration: $0.1 \mathrm{mV}$.

responses were evoked corepresented the same contralateral forelimb joint.

\section{ICMS-evoked forelimb responses correlate with EMG responses}

To ensure that evoked forelimb movements correlated with other measures of forelimb response to ICMS, we routinely used EMG recordings ( $n=8$ rats). The current threshold for evoking contralateral and ipsilateral joint movements corresponded closely to contralateral and ipsilateral agonist EMG thresholds, respectively. With increasing currents, contralateral EMG activity was more strongly modulated than ipsilateral EMG activity. The results from one representative control animal are presented in Figure 5. The threshold for evoking contralateral elbow flexion was $15 \mu \mathrm{A}$ and ipsilateral elbow flexion was $22 \mu \mathrm{A}$. Averaged EMG traces demonstrate a minimal contralateral response (Fig. $5 A$ ) at $20 \mu \mathrm{A}$ with progressively stronger responses with increasing stimulus strength. Figure $5 B$ shows a minimal ipsilateral response at $20 \mu \mathrm{A}$ and increasing responses up to $80 \mu \mathrm{A}$, which was the highest current tested. These results, confirmed in several animals at multiple cortical sites of varying threshold currents and in several muscle groups, validate the use of joint/limb movement in determining the response to ICMS. Furthermore, the findings support our observation that the ipsilateral response was less robust than the contralateral response. With higher currents, the increase in the contralateral response was greater than the increase in the ipsilateral response. Despite remarkable bilateral symmetry of the kind of the response, the magnitude of the ipsilateral responses was less than that of the contralateral response.

Evoked ipsilateral responses, but not contralateral responses, depend on the function of M1 in the other hemisphere We next determined whether the ipsilateral representation depended on the function of M1 in the other hemisphere and its associated descending brainstem and spinal pathways. To deter- 


\section{A. Ipsilateral responses}

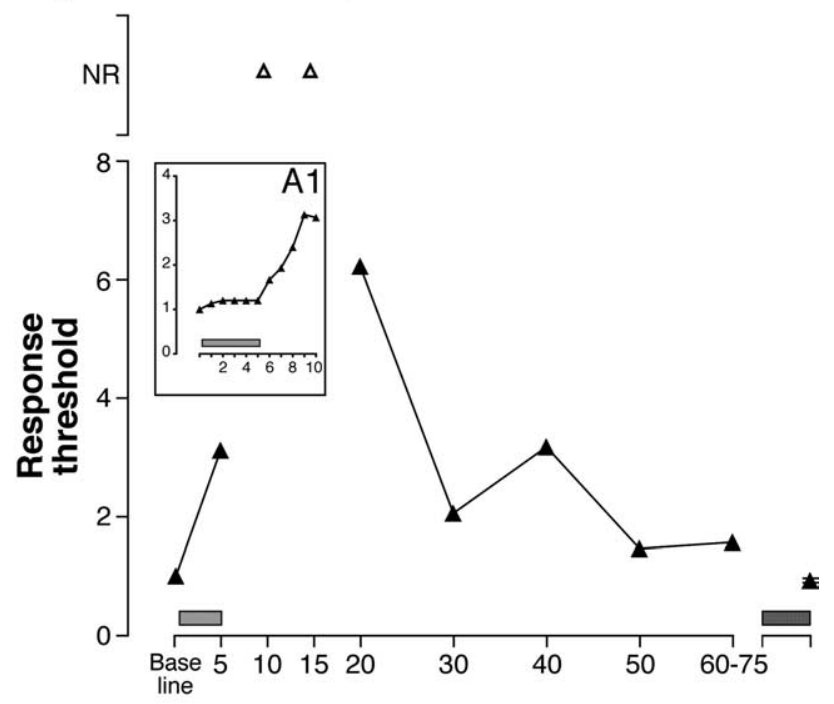

B. Contralateral responses

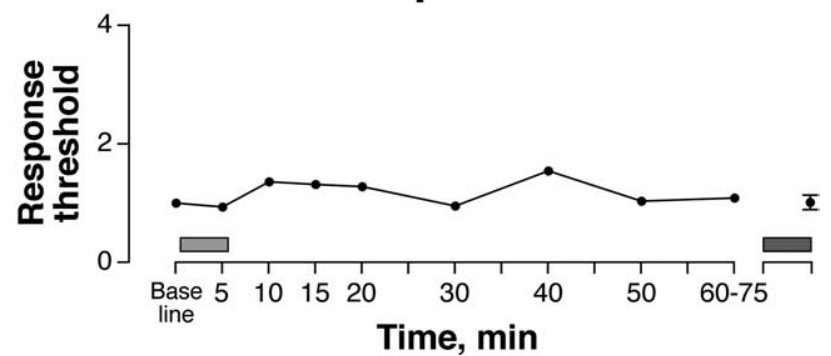

Figure 6. Ipsilateral forelimb responses require activity in the homotopic region of the other hemisphere. Time course of ipsilateral $(\boldsymbol{A})$ and contralateral $(\boldsymbol{B})$ responses after homotopic and nonhomotopic lidocaine injection into the forelimb area of M1 of the opposite hemisphere. Mean values of multiples of baseline response threshold are graphed for four animals. $\boldsymbol{A}_{\text {, Ho- }}$ motopic lidocaine inactivation reversibly blocked ipsilateral responses (triangles: closed, response evoked $>5-100 \mu \mathrm{A}$ range; open, response not evoked at $100 \mu \mathrm{A}$ ) by $10 \mathrm{~min}$, which returned to baseline by $50 \mathrm{~min}$. $\boldsymbol{A 1}$, Time course of lidocaine inhibition at $1 \mathrm{~min}$ increments in a single animal. $\boldsymbol{B}$, Contralateral responses are unaffected by inactivation of the other hemisphere (closed circles). The light gray bar below each graph immediately after the baseline value indicates the time period of the homotopic lidocaine injections. The dark gray bars on the right indicate the nonhomotopic infusion ( $n=2$, hindlimb inactivation; $n=1$, rostral forelimb representation).

mine the role of M1 function, we reversibly inactivated M1 in the nonstimulated hemisphere (see Fig. 1) by microinjection of the local anesthetic lidocaine. Lidocaine infusion ( $1 \mu \mathrm{l}$ of $2 \%$ solution over $5 \mathrm{~min}$ ) into the homotopic contralateral M1 rapidly and reversibly eliminated ipsilateral responses evoked from the noninactivated hemisphere, without affecting contralateral responses. Figure 6 plots the average multiple of response threshold elevation ( $n=4$ animals), for ipsilateral $(A)$ and contralateral $(B)$ responses. Ipsilateral response thresholds were elevated in each animal. In one animal we stimulated at $1 \mathrm{~min}$ intervals and observed that the threshold began to increase 5 min after injection onset, confirming prior observations that lidocaine injection rapidly inactivates neural tissue (Fig. 6A1) (Martin and Ghez, 2001). When thresholds rose $>100 \mu \mathrm{A}$, the site was considered unresponsive (Fig. 6A, NR). Although not systematically tested, we routinely used currents up to $150 \mu \mathrm{A}$ to verify the blockade. Ipsilateral response threshold began to return, on average, $20 \mathrm{~min}$ after injection onset and was within preinjection control values by $50 \mathrm{~min}$.
Thus, in M1 opposite to stimulation, lidocaine likely reduced the excitability of the most effective caudal M1 forelimb sites. Importantly, during the maximal block there was no significant elevation in threshold for evoking contralateral responses. Repeated-measures ANOVA indicated no change in the contralateral threshold throughout the time course of measurements $(F=0.74, p=0.69)$. This was also true during the time points of maximal ipsilateral inhibition (5-30 $\mathrm{min}, F=1.30, p=0.34$ ). Infusion at nonhomotopic sites (hindlimb area, $n=2$ rats; rostral forelimb subregion, $n=1$ rat) (Fig. 6, above gray bar) of the other hemisphere's M1 did not change the evoked contralateral ( $p=$ $0.39)$ or ipsilateral forelimb thresholds $(p=0.18)$.

The time course for lidocaine inactivation matches that of other reports (Martin and Ghez, 2001). The rapid reversal from inactivation to normal responding indicates that the elevation in threshold was not caused by the neural damage. Supplemental Figure 2, available at www.jneurosci.org as supplemental material, provides an estimate of lidocaine spread within M1. This amount of spread, $760 \mu \mathrm{m}$, is similar to a previous report using radiolabeled lidocaine with similar injection parameters (Martin, 1991). Importantly, it has been shown that drug spread associates closely with neural function. Glucose utilization is maximally suppressed the center of the lidocaine injection and returns to control levels as drug levels decrease (Martin, 1991). One microliter of $2 \%$ lidocaine reduced cortical neuronal activity with a radius of $630 \mu \mathrm{m}$ (Tehovnik and Sommer, 1997). When the area of lidocaine spread is overlaid on the average contralateral and ipsilateral forelimb response maps (supplemental Fig. 2 B, available at www.jneurosci.org as supplemental material), most of the sites in the caudal forelimb area with the strongest responses would be expected to be silenced.

Thus, inactivation of the M1 forelimb representation in the nonstimulated hemisphere reversibly elevated ipsilateral, but not contralateral, ICMS thresholds. Ipsilateral thresholds became elevated within minutes after the onset of the lidocaine injection, and returned shortly thereafter. This shows a dependence on the moment-to-moment function of the contralateral M1 for evoking ipsilateral responses. This dependence could reflect interhemispheric transfer via the corpus callosum (Fig. 1, thin blue line) or constitutive facilitation of brainstem or spinal cord motor circuits (Fig. 1, thick blue line). Importantly, the lack of an effect of M1 inactivation on the contralateral threshold suggests that callosal neurons do not constitutively regulate the excitability of M1 in the opposite hemisphere.

\section{Pyramidal tract lesion abolishes all responses evoked by ICMS from the damaged side and ipsilateral responses only from the undamaged side}

To determine whether descending projections from M1 are important for the expression of ipsilateral responses from M1 in the other hemisphere, we sectioned the PT on one side and mapped the motor representation in M1 on the opposite, intact, side (Fig. 1 , PT section). We recorded ipsilateral and contralateral motor responses from the hemisphere opposite PT section in 8 rats at various times after lesion (time after PT lesion: same day, $n=3$; $1 \mathrm{~d} ; n=1 ; 10 \mathrm{~d}, n=1 ; 2$ months, $n=2 ; 8$ months, $n=1$ ). Animals with PT lesion on the same day had the motor cortex mapped before and after PT section. Animals tested early ( 1 and $10 \mathrm{~d}$ ) after PT lesion were included to make sure that PT effects were not attributable to the prolonged deep anesthesia necessary for the PT surgery, or other acute effects of the surgery, in addition to the PT section. Animals tested after chronic injury (2 and 8 months) were included to test for postlesion plasticity, similar 
to adult control lesion experiments conducted by Kartje-Tillotson et al. (1985), who ablated M1 rather than the PT, and examined animals 4-5 months after lesion.

After the PT lesion, we reexamined at least five sites in the region of lowest ICMS thresholds (see Fig. 2). Animals that had the PT lesion on the same day both showed an elimination of ipsilateral responses in the region of the lowest thresholds for evoking ipsilateral responses before the lesion. Animals with $1 \mathrm{~d}-2$ month survival periods also showed the same effect, which was a complete absence of evoked ipsilateral responses from the intact M1. In the single animal examined 8 months after PT lesion, we replicated the Kartje-Tillotson et al. (1985) finding that ipsilateral responses recovered after damage of the CS system on the other side with longer survival time (supplemental Fig. 3, available at www.jneurosci.org as supplemental material). Thus, PT lesion, as reversible M1 inactivation, at all but $>4$ month survival times eliminated ipsilateral responses from the intact M1 providing further support for a dependence on the function of M1 in the other hemisphere. This result further suggests that the critical locus of interaction between the M1 outputs on the two sides is the spinal cord, because PT lesion spares cortical projections to brainstem nuclei.

In two animals examined on the day of the PT lesion, we also remapped M1 ipsilateral to the lesioned pyramid. We reexamined at least five sites in each animal where stimulation before the lesion evoked bilateral responses at the lowest thresholds. Confirming two prior studies (Z'Graggen et al., 2000; Piecharka et al., 2005), contralateral responses were abolished at currents up to $100 \mu \mathrm{A}$. Not reported in prior studies, we also found that ipsilateral responses from the damaged side were abolished with PT lesion. This shows that both the contralateral and ipsilateral motor responses evoked before lesion depend on the direct CST (Fig. 1, red).

\section{Lesions of M1 interhemispheric connections elevates the threshold of ipsilateral but not contralateral responses}

To determine whether stimulation evoked ipsilateral responses via an obligatory interhemispheric pathway, we tested the effect of corpus callosum section on ipsilateral response thresholds (Fig. 1, callosal section). Interhemispheric projections between $\mathrm{M} 1$ are reported to be located in the rostral corpus callosum, between +1.0 and $-1.5 \mathrm{~mm}$ relative to bregma (Bury and Jones, 2004). We verified this by making anterograde tracer injections into the center of the forelimb zone, identified by ICMS, in two animals. Figure $7 A$ shows labeled callosal axons in the intact corpus callosum in a control animal (A1, A2). Dense labeling (Fig. $7 A 1$, inset) extended from approximately 1.3 to $-0.5 \mathrm{~mm}$ anteroposterior, caudal to which labeling was very sparse (Fig. 7A2). After callosal section, no labeled axons were present contralateral to the injection (Fig. 7A3).

We determined the effect of lesion of interhemispheric M1 connections on ipsilateral and contralateral thresholds in 8 rats. Postmortem histological analyses revealed that four of these animals received complete corpus callosum lesions between its rostral pole (approximately $+1.8 \mathrm{~mm}$ ) to $-2 \mathrm{~mm}$ bregma. This is the region where the highest density of anterogradely labeled callosal fibers cross. Figure $6 \mathrm{~B}$ shows that, in these animals, callosal lesion significantly elevated the threshold of ipsilateral responses by $32.7 \%$ ( $p=0.036$ ). The other four rats had incomplete lesions, either with a portion of the rostral corpus callosum spared or the presence of labeled corpus callosal axons contralateral to the tracer injection. In these animals, callosal lesion resulted in an $18.5 \%$ increase in ipsilateral threshold, which was not significant $(p=0.43)$. For both groups combined, there was a significant $27.1 \%$ increase in ipsilateral threshold $(p=0.032)$. Whereas ipsilateral thresholds were elevated by callosal lesion, contralateral thresholds were unchanged $(p=0.49)$. That callosal section does not change contralateral thresholds indicates that in this preparation there is neither significant constitutive transcallosal excitation nor inhibition. This is consistent with the lack of change in contralateral threshold after M1 lidocaine inactivation, described earlier.

\section{Ipsilateral responses are restored after systemic 4-AP administration}

We next asked whether we could restore the capacity to evoke ipsilateral motor responses with ICMS after contralateral PT lesion $(n=3)$ and during reversible M1 inactivation $(n=2)$ by elevating CS system efficacy. The ipsilateral actions of the descending cortical projection are known to be weak (Edgley et al., 2004; Jankowska et al., 2005; Brus-Ramer et al., 2007). Following the lead of Jankowska et al. (2005) and Edgley et al. (2004), we reasoned that these connections could be made more effective by systemic administration of a $\mathrm{K}^{+}$channel blocker, 4-AP, which enhances synaptic transmission between descending cortical axons and brainstem and spinal targets, as well as generally increasing excitability (Jankowska et al., 1982).

We first determined the effect of 4-AP on ipsilateral responses in animals with PT lesion (i.e., lesion left PT; stimulate right M1) (see Fig. 1). We examined both acute PT lesion (same day, $n=2$; $1 \mathrm{~d}$ before testing, $n=1)$ and 2 months earlier $(n=2)$. We chose to examine the chronic postlesion animals because an earlier study showed that even at long survival times ipsilateral responses 
A. Baseline

A1. Ipsilateral

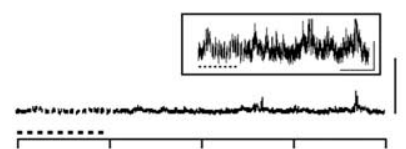

A2. Contralateral

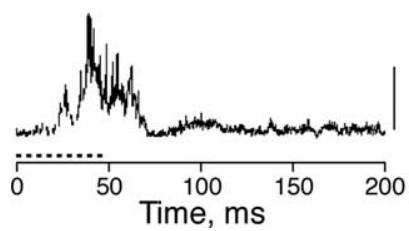

B. After 4-AP

B1. Ipsilateral

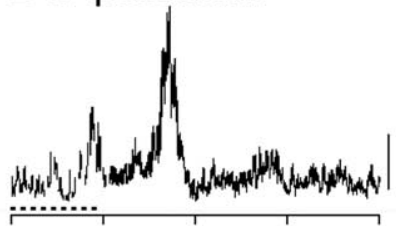

B2. Contralateral

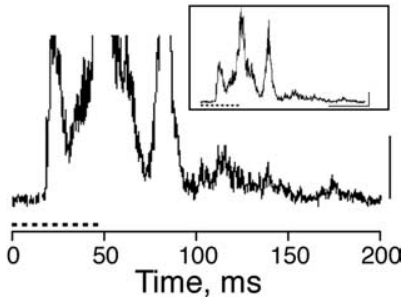

Figure 8. Effect of 4-AP on ipsilateral and contralateral biceps muscle activation after PT lesion. Ensemble averages of rectified and integrated biceps EMG ipsilateral $(\boldsymbol{A 1}, \boldsymbol{B} \mathbf{1})$ and contralateral $(\boldsymbol{A 2}, \boldsymbol{B} 2)$ to ICMS of the right M1 $(30 \mu \mathrm{A}) . \boldsymbol{A}$, EMG recordings before systemic administration of 4-AP. Inset in $A 1$ shows ipsilateral EMG at increased amplification. $\boldsymbol{B}$, EMG recordings after systemic administration of 4-AP. Inset in $B 2$ shows smaller image of contralateral EMG to reveal the peak responses, which were truncated in $\boldsymbol{B} 2$. Calibrations: $\boldsymbol{A 1}, \boldsymbol{B} 1, \boldsymbol{A} \mathbf{2}, \boldsymbol{B} 2,0.1 \mathrm{mV}$; inset $\boldsymbol{A 1}, 0.005 \mathrm{mV}$; inset $\boldsymbol{B} 2,0.1 \mathrm{mV}$.

cannot be evoked by M1 ICMS. Kartje-Tillotson et al. (1985) showed that before 4 months after PT lesion, stimulation of M1 on the intact side does not evoke ipsilateral responses. In these experiments, we made 5-10 penetrations within the region of lowest ICMS thresholds before 4-AP administration to determine the maximally effective sites in M1 for evoking forelimb responses. In the PT lesioned animals, contralateral responses were evoked at thresholds similar to those in intact animals and, in all cases, no ipsilateral responses were evoked. In these animals, increasing stimulation duration from 45 to $300 \mathrm{~ms}$ and amplitude to $150 \mu \mathrm{A}$ failed to evoke ipsilateral responses.

Figure 8 shows representative biceps EMG ensemble averages from an animal in which the PT was lesioned 2 months earlier. As expected, stimulation at the center of the forelimb zone before systemic 4 -AP injection ( $2 \mathrm{mg} / \mathrm{kg}$, i.p.) failed to evoke any ipsilateral responses at $100 \mu \mathrm{A}$ (A1), but did evoke contralateral biceps contraction at $30 \mu \mathrm{A}(\mathrm{A} 2)$. The inset in A1 shows the absence of an ipsilateral biceps response at higher amplification. Beginning 40 min after 4-AP injection, an ipsilateral biceps response was evoked at the same site by stimulation at $30 \mu \mathrm{A}(\mathrm{B} 1)$. 4-AP also evoked stronger contralateral response (B2; the peak EMG responses are truncated, but the inset shows the complete response at lower amplification). A similar potentiating effect of 4-AP on ipsilateral responding was observed for the other chronic and the acute PT lesioned animals.

We also determined whether 4-AP restored the capacity for evoking ipsilateral responses during reversible lidocaine inactivation of M1 on the nonstimulated side. We injected 4-AP at the same dose as above and waited $1 \mathrm{~h}$ before injecting lidocaine $(1 \mu \mathrm{l}$ of $2 \%$ lidocaine infused over $5 \mathrm{~min}$ ), to ensure that the time of maximal M1 blockade coincided with the actions of 4-AP. In one representative experiment, baseline ipsilateral ICMS threshold was $40 \pm 4.3 \mu \mathrm{A}$ and contralateral threshold, $19.5 \pm 2.6 \mu \mathrm{A}$. Fifty minutes after 4-AP administration, ipsilateral threshold was 33 $\mu \mathrm{A}$, and contralateral threshold was $25 \mu \mathrm{A}$. Ten and $15 \mathrm{~min}$ after the onset of lidocaine infusion into the nonstimulated M1 (i.e., as

Fig. 6), the ipsilateral threshold was $29 \mu \mathrm{A}$ and $35 \mu \mathrm{A}$, respectively. Contralateral threshold was $18 \mu \mathrm{A}$ at both times. Thus, M1 lidocaine inactivation, which normally completely blocked ipsilateral responses (Fig. 5), was ineffective in preventing ipsilateral responses after systemic 4-AP administration. During the same experiment, we repeated the lidocaine injection, with similar results (40 $\mu \mathrm{A}$ ipsilateral threshold $10 \mathrm{~min}$ after the second lidocaine injection). Comparable results were obtained in the second animal.

Two controls were done during these experiments. First, before 4-AP administration, we injected lidocaine and verified that this blocked ipsilateral responses, as above (Fig. 6). The 4-AP experiment was then conducted after a $1-1.5 \mathrm{~h}$ washout; the time depended on the return of thresholds to pre-inactivation levels. Second, we verified that M1 had been inactivated by stimulating near the lidocaine injection site, which failed to evoke any motor response. Our PT lesion and M1 inactivation experiments show that ipsilateral responses can be made to be independent of a functioning CS system in the other hemisphere by acutely augmenting the efficacy of CS system connections. The results suggest that latent ipsilateral connections could be used to access spinal motor circuits on the side of motor impairment soon after injury.

\section{Discussion}

The ipsilateral and contralateral M1 forelimb representations in rat mirror one another: The most effective sites for evoking contralateral responses were also the most effective for evoking ipsilateral responses. Moreover, the majority of individual sites corepresented the same sets of distal and proximal responses. Our findings provide further support for the idea that the ipsilateral CS system normally contributes to the on-going control of unimanual limb movements. Surprisingly, the ipsilateral representation normally depends on an intact CS system from the contralateral hemisphere. We found important interactions at both the interhemispheric level and below the site of PT lesion. While it is not possible to apportion these contributions quantitatively, our results point to the spinal cord as a key level of interaction because all ipsilateral responses were eliminated after pyramidotomy. Ipsilateral responses in M1 can be immediately reinstated when the efficacy of the CS system was augmented with 4-AP, suggesting a latent pathway to spinal motor control circuits (Jankowska and Edgley, 2006). Thus, the ipsilateral M1 motor representation and its descending connections could be an important component of recovery after unilateral pyramidal system damage.

\section{The ipsilateral M1 motor representation is efficient at evoking} both distal and proximal responses

Previous studies documented the presence of an ipsilateral motor map (Kartje-Tillotson et al., 1985, 1987; O’Donoghue et al., 1986; Liang et al., 1993). Ours is the first to systematically analyze the relation between ipsilateral and contralateral motor responses. The majority of sites where ipsilateral responses were evoked corepresented the same joint bilaterally and most ipsilateral responses occurred at the elbow or wrist, with remaining sites representing either more proximal or distal joints. Clearly, the ipsilateral representation is not limited to proximal control, as classical studies suggested (Woolsey et al., 1979). While not precluding a role in posture or proximal control, the ipsilateral CS projection also seems well suited for more distal control, as several human studies suggest (Jones et al., 1989; Wassermann et al., 1991, 1994; Bawa et al., 2004). In animals, ipsilateral CST con- 
nections are implicated in skilled locomotion (Loy et al., 2002) and have been shown to mediate distal control after injury (Weidner et al., 2001; Maier et al., 2008).

The ipsilateral representation is extensive and robust. It is nearly half the size of the contralateral representation and requires only one third higher stimulus strength to evoke responses. This is remarkable considering that the direct ipsilateral CST projection is so sparse, comprising only $5-6 \%$ of the contralateral projection (Brösamle and Schwab, 1997). There appears to be a gain in ability to activate ipsilateral spinal circuits disproportionate to its density. This gain in function could be achieved by having the contralateral CST from the other hemisphere tonically facilitate spinal motor circuits, as our lesion and inactivation findings suggest, or dynamically, by recruiting the opposite M1 via the corpus callosum. The ipsilateral representation could also be optimized through strengthening of local intracortical connections (Hess and Donoghue, 1994; Kleim et al., 2003) or descending brainstem connections (see below).

\section{Ipsilateral responses depend on the function of $\mathrm{M} 1$ in the opposite hemisphere}

Ipsilateral responses evoked by ICMS on the intact side were eliminated during inactivation of M1 in the other hemisphere or after lesion of the other PT. These results provoke the question of what are the key loci of convergence between the cortical motor systems on each side that enable M1 to express ipsilateral responses. ICMS can evoke ipsilateral responses via interhemispheric connections, corticofugal projections to the brainstem, and ipsilateral CST terminations. Interhemispheric convergence is important because callosal section significantly elevated ICMS thresholds; this is discussed further in the next section. PT lesion spares cortical projections to the brainstem while M1 inactivation does not, yet both abolish ipsilateral responses from the intact hemisphere. This suggests that convergence at the brainstem level is insufficient to maintain ipsilateral responses, and that the most important site of convergence is the spinal cord. Bilateral CST projections from the stimulated and unstimulated sides converge on common spinal motor circuits. Our results support a model whereby M1 control signals can evoke ipsilateral limb responses as long as spinal motor circuits are constitutively activated by projections from the homotopic opposite M1. The absence of effects of inactivating nonhomotopic regions further suggests that it is not simply global activity of the other hemisphere, but that a specific convergence pattern exists.

The idea that a specific bilateral convergence pattern at the spinal level is needed for effective ipsilateral control helps explain limited motor recovery in kittens that develop impaired CSTs on each side (Martin, 2005). Unilateral blockade of M1 activity during a critical period shifts contralateral CST terminations from more ventral to more dorsal spinal laminae. In contrast, the active CST in the opposite hemisphere develops a bilateral projection, with the aberrant ipsilateral terminations occupying the same territory as normally populated by the contralateral CST terminations (Friel and Martin, 2007; Salimi et al., 2008). Despite a dense ipsilateral projection to the intermediate zone and ventral horn, there is no ipsilateral M1 motor representation (Chakrabarty et al., 2007) and motor skills are impaired (Friel et al., 2007). In the context of our results in the mature rat, aberrant ipsilateral CST terminations in the kitten may not be able to express significant motor control functions because the contralateral CST projection to the ventral horn is weak. Our 4-AP data suggest that some of the need for this specificity may be overcome by a generalized elevation in CS system excitability
(Jankowska et al., 1982, 2005). However, while 4-AP could lead to expression of ipsilateral responding from M1, it might not lead to restoration of skilled movement control. The latter likely depends more on adaptive changes in particular circuits.

The role of M1 projections to brainstem motor control nuclei in mediating ipsilateral control is complex. Prior studies (Z'Graggen et al., 2000; Piecharka et al., 2005) and our findings suggest that cortical activation of brainstem motor pathways does not transmit ICMS-evoked signals, at least not soon after a unilateral lesion. Nevertheless, brainstem motor centers might be an important site of interaction. Jankowska, Edgley and colleagues have convincingly shown that augmenting the strength of cortical projections to reticulospinal neurons leads to stronger activation of ipsilateral motor pools (Edgley et al., 2004). Whereas this pathway may not be strong enough to evoke forelimb responses soon after PT lesion, it may help to restore ipsilateral responses after 4-AP or $>4$ months after PT lesion.

\section{What are the functions of interhemispheric signaling?}

Surprisingly, neither callosal section nor M1 inactivation changed the threshold for evoking contralateral responses. This suggests that callosal neurons in rat M1 do not exert significant tonic excitatory or inhibitory control. The lack of constitutive transcallosal inhibition appears to contrast with the prevailing view that callosal interactions in humans have a net inhibitory action (Ferbert et al., 1992; Meyer et al., 1995; Hanajima et al., 2001). However, excitatory homotopic and inhibitory heterotopic actions have been shown in cat M1 (Asanuma and Okuda, 1962). Stimulation of a restricted zone of homotopic M1 sites in one hemisphere evoked an excitatory response in CST neurons in the other, whereas stimulation of a large surrounding region evoked inhibition. This is like the center-surround organization of sensory receptive fields. Excitatory interhemispheric effects also have also been observed in humans using transcranial magnetic stimulation and a conditioning-test paradigm (Ugawa et al., 1993; Hanajima et al., 2001). In addition to being a potentially important site of interaction between the CS systems in each hemisphere, interhemispheric excitatory signaling could also be a key path for recruiting corticospinal and corticobulbar outputs from the opposite M1, via a double crossed pathway (Fig. 1).

\section{Can the ipsilateral CST control limb movements after unilateral pyramidal system damage in humans?}

The mirror organization of the contralateral and ipsilateral representations is well suited for contributing to recovery of distal and proximal control after unilateral CS system injury. However, the ipsilateral motor representation is normally dependent on a functioning representation in M1 of the opposite hemisphere, which likely limits the efficacy of spared ipsilateral projections after injury. This dependence on M1 of the opposite hemisphere can be overcome. When ipsilateral CST projections are made more effective with 4-AP [or the overall excitability of the motor systems is augmented (Nashmi and Fehlings, 2001)], during inactivation or soon after PT lesion, ipsilateral responses from M1 are immediately expressed. This suggests that latent connections with spinal circuits exist. Three potential loci for enhancing the efficacy of CST transmission at latent connections are synapses between callosal neuron terminals and M1 excitatory neurons, synapses between the CS system and brainstem motor centers, as stressed by Jankowska and Edgley (2006), and synapses between ipsilateral and double crossed CST spinal projections and spinal interneurons (Fig. 1). In addition to synapsing directly on motoneurons, in humans, the CST also projects to the intermediate 
zone (Schoen, 1964) and synapses on spinal interneurons (Pierrot-Deseilligny and Burke, 2005). Intrinsic segmental circuits could offer multiple paths for activating motoneurons.

It is plausible that taking advantage of such connections soon after an injury would be beneficial, especially since this early time window precedes the period when spasticity and spinal reflex changes emerge (Pierrot-Deseilligny and Burke, 2005; Nielsen et al., 2007). The dependence on M1 of the opposite hemisphere can also be overcome with time and without intervention. Four or more months after M1 lesion, during which time postlesion plasticity occurs (Castro, 1975), ipsilateral responses can be evoked from M1 on the intact side [(Kartje-Tillotson et al., 1985); supplemental Fig. 3, available at www.jneurosci.org as supplemental material]. Early activity-based interventions after unilateral pyramidal injury, such as CST activation (Brus-Ramer et al., 2007) and constraint-induced therapy (Maier et al., 2008), could strengthen ipsilateral CST terminations in the spinal cord, promote the ipsilateral M1 motor map, and help reinstate normal limb control.

\section{References}

Asanuma H, Okuda O (1962) Effects of transcallosal volleys on pyramidal tract cell activity of cat. J Neurophysiol 25:198-208.

Bawa P, Hamm JD, Dhillon P, Gross PA (2004) Bilateral responses of upper limb muscles to transcranial magnetic stimulation in human subjects. Exp Brain Res 158:385-390.

Brösamle C, Schwab ME (1997) Cells of origin, course, and termination patterns of the ventral, uncrossed component of the mature rat corticospinal tract. J Comp Neurol 386:293-303.

Brus-Ramer M, Carmel JB, Chakrabarty S, Martin JH (2007) Electrical stimulation of spared corticospinal axons augments connections with ipsilateral spinal motor circuits after injury. J Neurosci 27:13793-13801.

Bury SD, Jones TA (2004) Facilitation of motor skill learning by callosal denervation or forced forelimb use in adult rats. Behav Brain Res 150:43-53.

Castro AJ (1975) Ipsilateral corticospinal projections after large lesions of the cerebral hemisphere in neonatal rats. Exp Neurol 46:1-8.

Chakrabarty S, Friel KM, Martin JH (2009) Activity-dependent plasticity improves M1 motor representation and corticospinal tract connectivity. J Neurophysiol 101:1283-1293.

Cisek P, Crammond DJ, Kalaska JF (2003) Neural activity in primary motor and dorsal premotor cortex in reaching tasks with the contralateral versus ipsilateral arm. J Neurophysiol 89:922-942.

Donchin O, Gribova A, Steinberg O, Mitz AR, Bergman H, Vaadia E (2002) Single-unit activity related to bimanual arm movements in the primary and supplementary motor cortices. J Neurophysiol 88:3498-3517.

Edgley SA, Jankowska E, Hammar I (2004) Ipsilateral actions of feline corticospinal tract neurons on limb motoneurons. J Neurosci 24:7804-7813.

Ferbert A, Priori A, Rothwell JC, Day BL, Colebatch JG, Marsden CD (1992) Interhemispheric inhibition of the human motor cortex. J Physiol 453:525-546

Friel KM, Martin JH (2007) Bilateral activity-dependent interactions in the developing corticospinal system. J Neurosci 27:11083-11090.

Friel KM, Drew T, Martin JH (2007) Differential activity-dependent development of corticospinal control of movement and final limb position during visually-guided locomotion. J Neurophysiol 97:3396-3406.

Hanajima R, Ugawa Y, Machii K, Mochizuki H, Terao Y, Enomoto H, Furubayashi T, Shiio Y, Uesugi H, Kanazawa I (2001) Interhemispheric facilitation of the hand motor area in humans. J Physiol 531:849-859.

Hess G, Donoghue JP (1994) Long-term potentiation of horizontal connections provides a mechanism to reorganize cortical motor maps. J Neurophysiol 71:2543-2547.

Jankowska E, Edgley SA (2006) How can corticospinal tract neurons contribute to ipsilateral movements? A question with implications for recovery of motor functions. Neuroscientist 12:67-79.

Jankowska E, Lundberg A, Rudomin P, Sykova E (1982) Effects of 4-aminopyridine on synaptic transmission in the cat spinal cord. Brain Res 240:117-129.

Jankowska E, Cabaj A, Pettersson LG (2005) How to enhance ipsilateral actions of pyramidal tract neurons. J Neurosci 25:7401-7405.
Jones RD, Donaldson IM, Parkin PJ (1989) Impairment and recovery of ipsilateral sensory-motor function following unilateral cerebral infarction. Brain 112:113-132.

Kartje-Tillotson G, Neafsey EJ, Castro AJ (1985) Electrophysiological analysis of motor cortical plasticity after cortical lesions in newborn rats. Brain Res 332:103-111.

Kartje-Tillotson G, O’Donoghue DL, Dauzvardis MF, Castro AJ (1987) Pyramidotomy abolishes the abnormal movements evoked by intracortical microstimulation in adult rats that sustained neonatal cortical lesions. Brain Res 415:172-177.

Kleim JA, Bruneau R, Calder K, Pocock D, VandenBerg PM, MacDonald E, Monfils MH, Sutherland RJ, Nader K (2003) Functional organization of adult motor cortex is dependent upon continued protein synthesis. Neuron 40:167-176.

Liang F, Rouiller EM, Wiesendanger M (1993) Modulation of sustained electromyographic activity by single intracortical microstimuli: comparison of two forelimb motor cortical areas of the rat. Somatosens Mot Res $10: 51-61$.

Loy DN, Talbott JF, Onifer SM, Mills MD, Burke DA, Dennison JB, Fajardo LC, Magnuson DS, Whittemore SR (2002) Both dorsal and ventral spinal cord pathways contribute to overground locomotion in the adult rat. Exp Neurol 177:575-580.

Maier IC, Baumann K, Thallmair M, Weinmann O, Scholl J, Schwab ME (2008) Constraint-induced movement therapy in the adult rat after unilateral corticospinal tract injury. J Neurosci 28:9386-9403.

Marshall RS, Perera GM, Lazar RM, Krakauer JW, Constantine RC, DeLaPaz RL (2000) Evolution of cortical activation during recovery from corticospinal tract infarction. Stroke 31:656-661.

Martin JH (1991) Autoradiographic estimation of the extent of reversible inactivation produced by microinjection of lidocaine and muscimol in the rat. Neurosci Lett 127:160-164.

Martin JH (2005) The corticospinal system: from development to motor control. The Neuroscientist 11:161-173.

Martin JH, Ghez C (2001) Dissecting the organization of cortical and subcortical circuits for skilled limb movement control in development and maturity with reversible inactivation. In: Virtual lesions: understanding behavior and perception with reversible deactivation techniques (Lomber S, Galuske R, eds). Oxford: Oxford UP.

Meyer BU, Röricht S, Gräfin von Einsiedel H, Kruggel F, Weindl A (1995) Inhibitory and excitatory interhemispheric transfers between motor cortical areas in normal humans and patients with abnormalities of the corpus callosum. Brain 118:429-440.

Nashmi R, Fehlings MG (2001) Mechanisms of axonal dysfunction after spinal cord injury: with an emphasis on the role of voltage-gated potassium channels. Brain Res Brain Res Rev 38:165-191.

Neafsey EJ, Bold EL, Haas G, Hurley-Gius KM, Quirk G, Sievert CF, Terreberry RR (1986) The organization of the rat motor cortex: a microstimulation mapping study. Brain Res 396:77-96.

Nielsen JB, Crone C, Hultborn H (2007) The spinal pathophysiology of spasticity-from a basic science point of view. Acta Physiol (Oxf) 189:171-180.

O’Donoghue DL, Kartje-Tillotson G, Neafsey EJ, Castro AJ (1986) A study of forelimb movements evoked by intracortical microstimulation after hemicerebellectomy in newborn, young and adult rats. Brain Res 385:311-320.

Paxinos G, Watson C (1986) The rat brain in stereotaxic coordinates, Ed 2. Sydney: Academic.

Piecharka DM, Kleim JA, Whishaw IQ (2005) Limits on recovery in the corticospinal tract of the rat: partial lesions impair skilled reaching and the topographic representation of the forelimb in motor cortex. Brain Res Bull 66:203-211.

Pierrot-Deseilligny E, Burke D (2005) The circuitry of the human spinal cord. Cambridge, UK: Cambridge UP.

Salimi I, Friel KM, Martin JH (2008) Pyramidal tract stimulation restores normal corticospinal tract connections and visuomotor skill after early postnatal motor cortex activity blockade. J Neurosci 28:7426-7434.

Sanes JN, Wang J, Donoghue JP (1992) Immediate and delayed changes of rat motor cortical output representation with new forelimb configurations. Cereb Cortex 2:141-152.

Schaefer SY, Haaland KY, Sainburg RL (2007) Ipsilesional motor deficits following stroke reflect hemispheric specializations for movement control. Brain 130:2146-2158. 
Schoen JH (1964) Comparative aspects of the descending fibre systems in the spinal cord. Prog Brain Res 11:203-222.

Tehovnik EJ, Sommer MA (1997) Effective spread and timecourse of neural inactivation caused by lidocaine injection in monkey cerebral cortex. J Neurosci Methods 74:17-26.

Ugawa Y, Hanajima R, Kanazawa I (1993) Interhemispheric facilitation of the hand area of the human motor cortex. Neurosci Lett 160:153-155.

Wang J, Sainburg RL (2006) The symmetry of interlimb transfer depends on workspace locations. Exp Brain Res 170:464-471.

Ward NS, Cohen LG (2004) Mechanisms underlying recovery of motor function after stroke. Arch Neurol 61:1844-1848.

Wassermann EM, Fuhr P, Cohen LG, Hallett M (1991) Effects of transcranial magnetic stimulation on ipsilateral muscles. Neurology 41:1795-1799.
Wassermann EM, Pascual-Leone A, Hallett M (1994) Cortical motor representation of the ipsilateral hand and arm. Exp Brain Res 100:121-132.

Weidner N, Ner A, Salimi N, Tuszynski MH (2001) Spontaneous corticospinal axonal plasticity and functional recovery after adult central nervous system injury. Proc Natl Acad Sci U S A 98:3513-3518.

Woolsey CN, Erickson TC, Gilson WE (1979) Localization in somatic sensory and motor areas of human cerebral cortex as determined by direct recording of evoked potentials and electrical stimulation. J Neurosurg 51:476-506.

Z'Graggen WJ, Fouad K, Raineteau O, Metz GA, Schwab ME, Kartje GL (2000) Compensatory sprouting and impulse rerouting after unilateral pyramidal tract lesion in neonatal rats. J Neurosci 20:6561-6569. 